\title{
Design on Spaceborne Three Orthotropic Thin-Wall Tubular Antennas Deployment Mechanism
}

\author{
Wenjie Fan ${ }^{1}$, Lin Wu ${ }^{1}$, Hongmin Pen ${ }^{2}$ \\ ${ }^{1}$ Key Laboratory of Microwave Remote Sensing, National Space Science Center, Chinese Academy of Sciences, Beijing 100190, China \\ 2 Tianjin Aerospace Electromechanical Equipment Research Institute, Tianjin 300458, China
}

\begin{abstract}
The purpose of spaceborne three orthotropic thin-wall tubular antenna is for space science probe which consists of three orthotropic tubular antennas. Three orthotropic thin-wall tubular antennas deployment mechanism is designed which consists of upper plate, lower plate, reel, burn-wire and tension spring. Flattened antenna tube can be wound in a reel which can be hold-down and locked by Dyneema string. Release by giving current to resistor, the string will melt while its temperature reaches its burning point, then the upper plate will be pulled up and the antennas can be deployed by stored strain energy in itself. According to a certain project requirements three orthotropic thinwall tubular antennas mechanism prototype was developed and tested, antenna tube was broken due to "blossom" phenomenon in deployment process. For avoiding "blossom" during deployment of antennas mechanism, the flange of reel is improved, so antennas can be deployed smoothly. Compared to motor-driven antenna mechanism, this antennas deployment mechanism has advantages of simple structure, lower mass and lower power consumption.
\end{abstract}

\section{Introduction}

Deep space exploration has became frontier field competed in space countries in 21 st century, while space distribution longest wavelength radio interference imaging can offer the unique observation data for it. Low-frequency radio spectrometer has three meter-class metallic antennas which are orthotropic in space. To achieve exploration objective of space science and meet requirements of the envelope of rocket and limits of mass and power, the exploration antenna should meet requirements of wide frequency, low mass and volume, low power, easy mounting, high and low temperature proof and deployable capacity.

The three orthotropic antennas in this article adopts thinwalled tubular antennas which have simple structure, high deployment reliability and belong to one dimension space deployable mast[1-4]. At present, research on thinwalled tubular antenna mainly focus on root drum model(RDM) - the rotating drum remains at the base of the unit during extension. Usually RDM use a motor for the purpose of retraction; the motor also can be used to control extension velocity, but its mass, volume and power is high and can't be used on micro-satellite. Research on multi-deployment mast mainly concentrate on the deployment masts lie in one plane and they are all wound on one drum deployed by one motor[3-5], so it can't be used in orthotropic antennas which don't lie in one plane.

Three orthotropic antennas deployment mechanism is designed which is comprised of upper plate, lower plate, drum, burning wire and tension spring. The tubular antenna is flattened into a flat tape, then coiled onto a drum for storage, so it contain stored elastic energy. The upper plate, the lower plate and Dyneema string can be used to hold and lock the drum. Give current to resistor for releasing antenna, the burn wire melt when the temperature reach the melting point, then the upper plate is pull up and the antenna deploys with the drum under itself elastic energy. The three orthotropic antennas deployment mechanism not only has lower extension velocity, low shock, but also avoid blossom phenomenon which can destroy antenna tube. The three orthotropic antennas deployment mechanism has low volume, low mass and low power, also complete vibration and thermal test.

\section{Design of three orthotropic antennas deployment mechanism}

To achieve space science exploration objective, exploration antennas are full polarization and three orthotropic style which are comprised of three pairwise orthogonal antennas. Fig.1 shows antenna deployment mechanism in deployed status. Due to envelope limit of rocket, antennas should have retraction capacity. Slit thin-walled tubular antennas have many cross section which are overlapping single element, overlapping double element, inter-locking double element and lenticular welded double element, and each has its advantages and disadvantages. The process of inter- 
locking double element is complicated; overlapping double element needs two drums to retract two antennas and has big volume; the lenticular welded double element has high torsional stiffness, but the process isn't good for metal and the fundamental modal of antenna is bending mode. Overlapping single element has simple structure, perfect process, low mass and low volume, so it is chosen for three orthotropic antennas. The cross-section of overlapping single element is shown in Fig. 2. There is a part of overlap in this kind of antenna and this makes antenna has better stiffness.

As is shown in Fig.3, three orthotropic antennas are comprised of three slit thin walled antennas independently and mounted in one base which mounted on a box that printed circuit board is in it. There are three pillars on the box and one axis is between each two pillars. The upper plate is mounted on one axis and rounds on it, but lower plate is fixed on the base. Fig.4 shows magnification of local of antenna deployment mechanism. It can be seen that three antenna are flatten and wound on three drums, however the other side of each antenna is fixed. Teeth were set on the upper plate and drum for gearing and constrain the rotational degree of freedom, and the lower plates are constrained in the axial. The Dyneema strings go through the holes of upper plates and lower plates for pushing down the drums tightly. Thus the 6 dof are constrained for hold down and lock. Give current to the resistors for heating and melting the strings. The upper plates are pull up after the strings are melt, then the antennas deploy by the elastic strain energy. For high reliability, a spring-pull pillar is used for kick off.

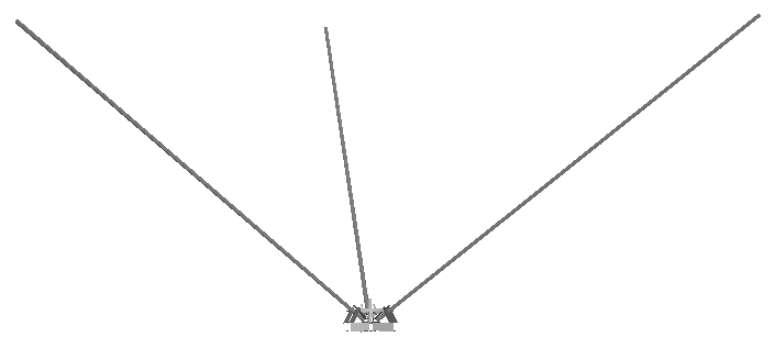

Figure 1. Antenna deployment mechanism in deployed status

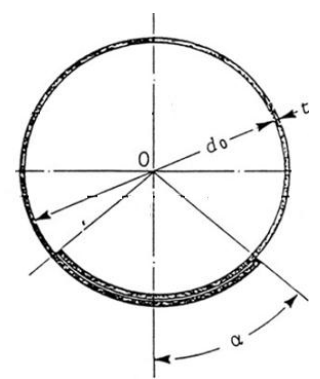

Figure 2. Section of antenna tube

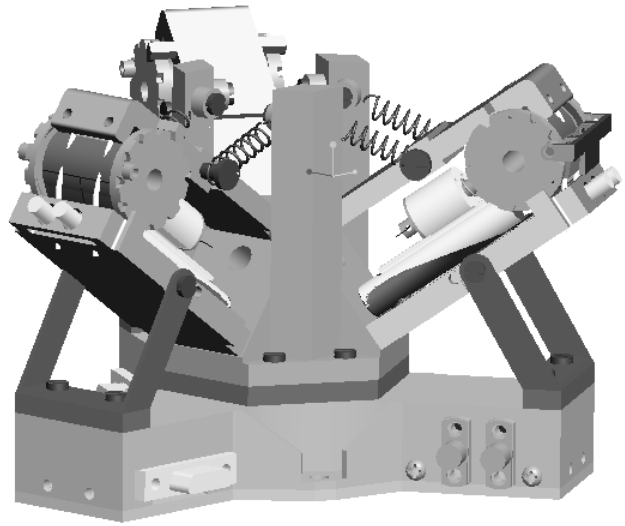

Figure 3. Deployment Mechanism of antenna in stowed status

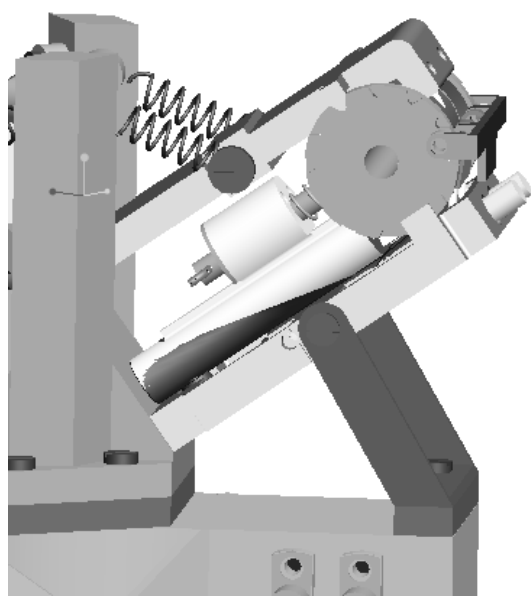

Figure 4. Local magnification of deployment mechanism

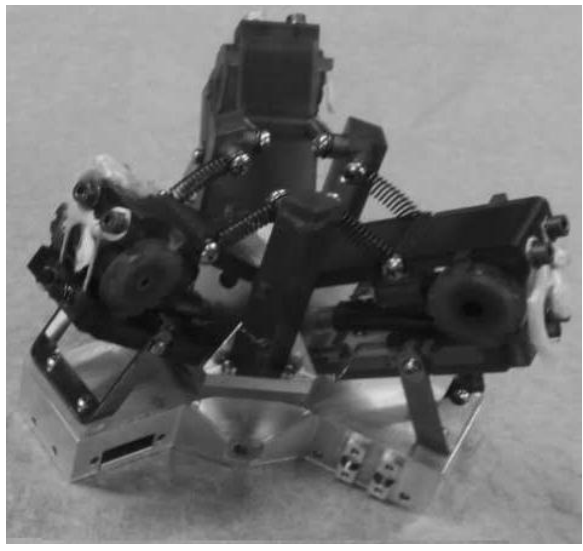

Figure 5. Product of mechanism

\section{Example for three orthotropic antennas deployment mechanism}

\author{
3.1 Three orthotropic antennas deployment \\ mechanism
}


Detection frequency of payload is $1 \sim 30 \mathrm{MHz}$, so the antenna is 1 meter long, $0.05 \mathrm{~mm}$ thick and has $6 \mathrm{~mm}$ in diameter. The overlap angle is $77.5^{\circ}$ and the mass is only $10 \mathrm{~g}$ for one antenna. The antennas are flatten and wound on the drums. The drums move with deployment of the antennas and can reach higher speed at the end of deployment. The drums are connected in the antennas with strings so that the drums don't become debris in space. Fig. 5 shows the product of three orthotropic tubular antennas deployment mechanism in stowed status. This kind of antenna deployment mechanism has high stow ratio. The envelope is $110 \mathrm{~mm} \times 110 \mathrm{~mm} \times 75 \mathrm{~mm}$ in stowed status and $1740 \mathrm{~mm} \times 1740 \mathrm{~mm} \times 620 \mathrm{~mm}$ in deployed status. The antennas are made of beryllium cooper, as it has not only high yield strength but also perfect electromagnetic properties. The upper plates and lower plates are made of polyimide. Compare to motordriven concept, it's lighter, smaller and little power.

\subsection{Deployment test and structure improvement for drum}

For validating performance of antenna deployment mechanism, single antenna deployment test are performed before three orthotropic antennas deploy. Antenna tube was broken during deployment, as is shown in Fig.6. The cause of fracture of antenna tube in the initial deployment phase are analyzed and the drum didn't deploy with antenna tube, so 'blossom' phenomenon happened in antenna tube, as is shown in Fig.6(a). Thus the drum can't deploy with antenna tube, so it bend under gravity, as is shown in Fig.6(b). So avoiding 'blossom' phenomenon is the key element for deployment successfully.
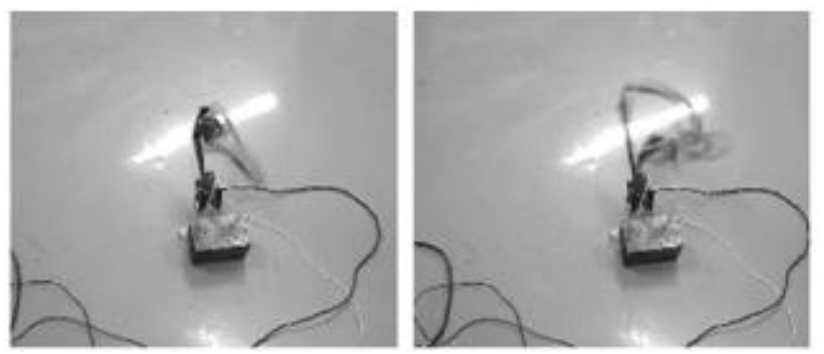

(a)
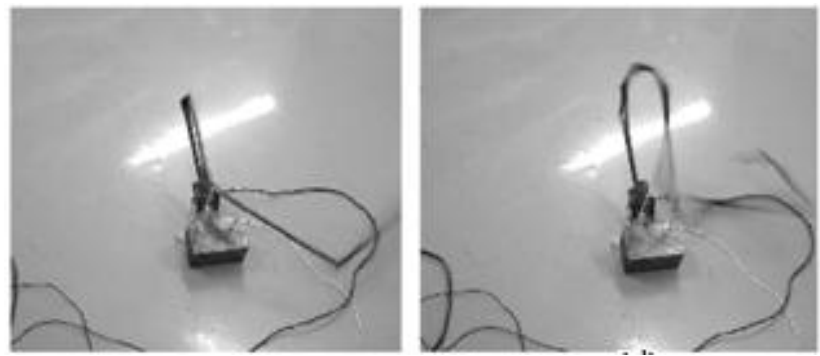

(b)
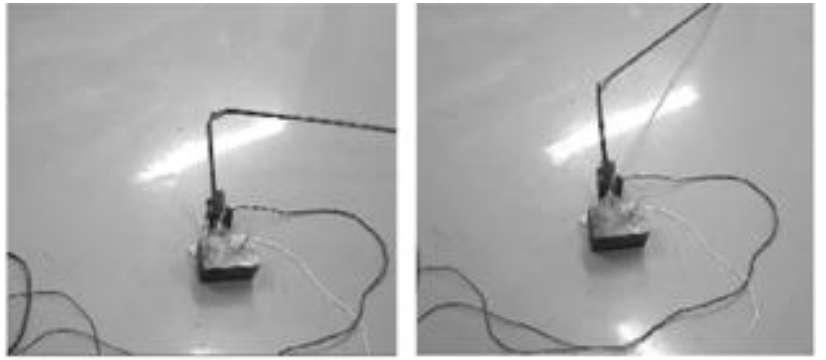

(c)

Figure 6. Deployment test of single antenna

Fig.7(a) shows drum before improvement and the two sides of drum are straight plates, so antenna tube can't be constrained in the deployment that swelled by itself elastic strain energy and bend under gravity. For solve this problem, the two sides of drum is improved into flanges which seem like concave plate. Thus they can constrain antenna tube for avoiding swell, as is shown in Fig.7(a). It was proved effective in deployment.

Three orthotropic antennas deployment test was performed successfully after single antenna tube deployed perfectly. But there is still a shortcoming in deployment time of three antennas. It can be deduced that heater mounting style and burn-wire winding style are the main reason that make the differences in deployment time. Fig. 8 shows the three Orthotropic Tubular Antennas Deployment Mechanism in deployed status.
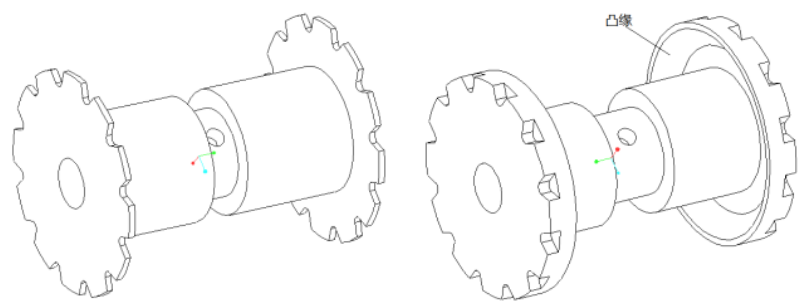

Figure 7. Structure of reel before and after improvement

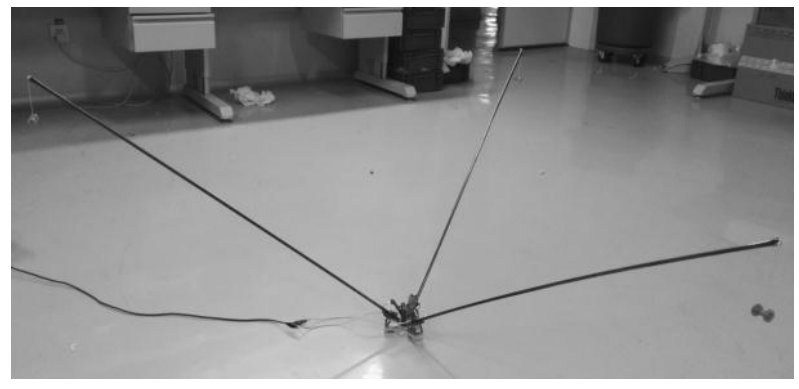

Figure 8. Three Orthotropic Tubular Antennas Deployment Mechanism in deployed status

\section{Conclusion}

Three orthotropic antennas deployment mechanism are developed which are comprised of upper plate, lower plate, drums and burn wire release element. There is a spring pre-loaded pull-bar for kick off for high reliability. The product is developed and deployment test is 
performed which proved deployment performance. Improvement on drum solve the problem that blossom phenomenon at the first of deployment, so the three antenna tubes can deploy smoothly and it also can decrease the speed by friction. It has smaller volume, lighter weight and lower power. The concept on antenna deployment mechanism can be applied in monopole and dipole antennas.

\section{References}

1. Gunnar Tibert. Deployable Tensegrity Structures for Space Applications[D].Royal Institute of Technology, Stockholm,Sweden,2002:10-16

2. Marco Straubel. Design and Sizing Method for Deployable Space Antennas[D]. Otto-von-Guericke University, Magdeburg, Germany,2012:14-21

3. Herzel, George G., Principal Contributor, "Tubular Spacecraft Booms (Extendible, Reel Stored)"'[R], NASA SP 8065, Feb. 1971.

4. Chen liemin, et al. Space structure and mechanism[M]. Beijing: China Science and Technology Press, 2005:314-321(in Chinese)

5. J.M. Fernandez, M. Schenk, G. Prassinos, V.J. Lappas, S.O. Erb. Deployment Mechanisms of
Gossamer Satellite Deorbiter[C]. 15th European Space Mechanisms \& Tribology Symposium, ESMATS 2013 Noordwijk, The Netherlands, 25-27 September 2013

6. Fan Wenjie, Wu Lin. Design and mechanics Analysis on a X-type Cross-section Tubular Boom[J]. Chinese Journal of Space Science, Vol.(37)5:616621(in Chinese)

7. MARCO Straubel, JOACHIM Block, MICHAEL Sinapius, et al. Deployable composite booms for various gossamer space structures[C]. The 52nd AIAA/ASME/ASCE/AHS/ASC Structures. Structural Dynamics and Materials Conference. Denver, Colorado, AIAA,2011

8. Hereck, L., et al. Development and test of deployable ultra-lightweight cfrp-booms for a solar sail[C]. European Conference on Spacecraft Structures, Material and Mechanical Testing 2002, CNES. Toulouse, France

9. F.P.J.RIMROTT, G.FRITZSCHE. Fundamentals of STEM Mechanism[C]. IUTAM-IASS Symposium on Deployable Structures: Theory and Applications, 2000:321-333 